\title{
Mejora en la satisfacción del cliente bajo el enfoque de la lógica borrosa
}

\section{Improvement in customer satisfaction under the approach of blurred logic}

\author{
José Néstor Ochoa Rodríguez ${ }^{1 *}$, Kléber Antonio Luna Altamirano ${ }^{1}$, Juan Carlos Erazo Álvarez ${ }^{1}$ y William \\ Henry Sarmiento Espinoza ${ }^{1}$ \\ ${ }^{1}$ Universidad Católica de Cuenca \\ *jochoa5@hotmail.com
}

DOI: https://doi.org/10.26871/killkana_social.v3i1.378

\begin{abstract}
Resumen
Lograr la satisfacción del cliente, es un problema latente en las instituciones del sistema financiero ecuatoriano, muy en especial en las agencias del Banco Pichincha de la ciudad de Cuenca-Ecuador, por ello el objetivo de la presente investigación es detectar las acciones y efectos, con el propósito de tratar de dar solución a este problema a través de variables escondidas que fueron omitidas o no consideradas por los expertos funcionarios de esta entidad bancaria. Dentro de la metodología se detalla el desarrollo de estas herramientas de avanzada que proporciona la lógica borrosa. Se emplea la técnica del expertizaje con el propósito de reducir la incertidumbre, esta sirve como mecanismo base para la elaboración de una matriz cuadrada de efectos olvidados, en donde se emplea el proceso de convolución max-min, para encontrar las variables escondidas u omitidas. De esta aplicación resulta como efecto olvidado el Incremento en la confianza institucional, si los directivos toman en consideración este efecto, lograrán mejorar la satisfacción al cliente.
\end{abstract}

Palabras clave: Efectos olvidados, expertizaje, lógica borrosa, satisfacción del cliente.

\begin{abstract}
Achieving customer satisfaction is a latent problem in the institutions of the Ecuadorian finantial system, especially in the "Banco del Pichincha" bank agencies in Cuenca-Ecuador. Therefore, the objective of this research is to detect actions and effects, with the purpose of attempting to solve this problem by using hidden variables that were either omitted or not considered by the expert officials of this bank. As part of the methodology, the development of advanced fuzzy logic tools is detailed. The expertise technique is implemented with the purpose of reducing uncertainty; this works as a basic mechanism for developing a square matrix of forgotten effects, where the process of max-min convolution is used to find the hidden or omitted variables. The forgotten effect obtained when applying this methodology results in an increase in Institutional trustworthiness. Therefore, if the managers take this effect into consideration, they will improve customer satisfaction
\end{abstract}

Keywords: Forgotten effects, expertise, fuzzy logic, customer satisfaction.

\section{Introducción}

La satisfacción del cliente es haber cumplido las expectativas con relación al servicio o producto que recibe el usuario, el no cumplimiento de esta acción se convierte en el problema que tiene el Banco Pichincha de la ciudad de Cuenca-Ecuador, por lo que a través de este estudio se trata de resolver este inconveniente que atraviesa esta institución financiera. Para ello se requiere apoyo de los expertos funcionarios, quienes entregan sus conocimientos relacionados a las acciones y efectos con la finalidad de generar una nueva estrategia de gestión para afrontar este problema y tratar de resolverlo.
La presente investigación da a conocer el desarrollo de herramientas de vanguardia que brinda la lógica difusa conocida también como lógica borrosa. Estas teorías son el expertizaje y efectos olvidados, impulsados y desarrollados por Kaufmann y Gil Aluja (1989), con el propósito de descubrir variables que se encuentran ocultas y que no han sido observadas por parte de los expertos funcionarios de la entidad bancaria. Estas deben considerarse para tratar de mejorar la satisfacción del cliente, con ello la imagen institucional será identificada y distinguida ante el resto de instituciones financieras del país. Dentro de la metodología se explica detenidamente la operatividad de estas 
herramientas. El objetivo de este estudio, es encontrar la vía correcta a través del desarrollo y aplicación de estas herramientas de vanguardia con la finalidad de reducir la incertidumbre mediante efectos olvidados, y que deben ser tomados en consideración por la institución financiera para lograr un adecuado servicio de atención al cliente que garantice su satisfacción.

\section{Estado del arte}

Lograr la satisfacción del cliente mediante el cumplimiento de sus necesidades y expectativas, es un reto de toda organización, por ello ciertos autores dan a conocer sus estudios relacionados con este problema: Romero y Romero (2006) realizan un análisis para determinar la satisfacción de los clientes externos con relación a los servicios prestados por las empresas aseguradoras en el municipio de Maracaibo Venezuela. Pérez, Martínez, Noda y de Miguel (2015) efectúan un análisis de la relación que existe entre la calidad ofrecida por el destino turístico de Holguín, y la satisfacción de los turistas, para este análisis emplean la estadística multivariada. Galvis y Ortiz (2011) generan la medición de la satisfacción de clientes en clínicas y hospitales de Bucaramanga y área metropolitana, con el propósito de identificar la gestión que realizan las clínicas y hospitales en cuanto a la medición de la satisfacción de sus clientes. Hernández, Medina y Hernández (2014) proponen un procedimiento para la medición y evaluación de la satisfacción de los clientes externos en empresas de base tecnológica de producciones por proyecto único o singular. Castrillón y Díaz (2010) establecen un modelo que permite descomponer el concepto en dimensiones del servicio y estas a su vez en indicadores, para esto emplean la metodología de modelización mediante sistemas de ecuaciones estructurales. Vela (2010) indica que la satisfacción al cliente es uno de los temas más importantes en la actualidad científica del marketing, por lo que para esta investigación se centra en el análisis del modelo de satisfacción propuesto por Oliver (1980) con la ayuda de la escala de Avkiran (1999), con personas de origen latinoamericano o canadiense. Almaguer, Pérez y Pérez (2014) desarrollan un procedimiento para la mejora de la satisfacción de los clientes en el hotel Miraflores de la cadena Islazul basado en los postulados de la lógica difusa.

Algunos autores han entregado su aporte con respecto a la teoría de efectos olvidados, como herramienta de vanguardia de la lógica difusa, llamada también lógica borrosa, entre ellos: Gento, Lazzari y Machado (2001) dan a conocer sus experiencias en la aplicación de la metodología de recuperación de efectos olvidados en diferentes problemas de gestión, presentan algunas reflexiones sobre su utilización, sobre los efectos de orden mayor que dos, acerca de la incidencia del tiempo si se considera un proceso dinámico, a más de ello definen la estabilidad estricta y no estricta de una matriz de incidencia. Rico y Tinto (2010) proponen la utilización de herramientas desarrolladas con base en la teoría de los subconjuntos borrosos, como el expertizaje- contraexpertizaje, y la teoría de los efectos olvidados en el tratamiento ex post de la información contable tradicional, con el fin de mejorar su capacidad para sustentar la toma de decisiones adecuadas a mediano y largo plazo.

Por su parte, Salazar (2012) desarrolla un modelo no lineal para la predicción del comportamiento del tipo de cambio a futuro basado en la opinión de expertos, estas opiniones son tratadas mediante la teoría de efectos olvidados de la lógica borrosa. Tinto, Luna y Cisneros (2017) explican la teoría de efectos olvidados a través de variables escondidas que no son fáciles de detectar por el artesano y que deben tomarse en cuenta, ya que afectan la comercialización y permiten el rescate de esta actividad. Aguiar (2004) en su investigación expresa que las situaciones de incertidumbre se identificarían, en cambio, por el hecho de que no solo desconocemos el resultado final, sino que no podemos predecirlo tampoco en términos de probabilidades objetivas.

Otros autores han dado un aporte a sus investigaciones a través de la lógica difusa. Kaufmann y Gil (1987) quienes aportaron con su valioso conocimiento de la lógica difusa a través de su obra Técnicas Operativas de Gestión para el Tratamiento de la Incertidumbre, definiendo a un número borroso como una secuencia finita o infinita de intervalos de confianza. Reig y González (2002) afirman: "la lógica borrosa se revela como un instrumento muy potente $(. .$. al permitir, por un lado, recoger la incertidumbre generada por el entorno de la empresa, y por otro tratar la subjetividad que implica toda opinión de expertos" (p.436). Kaufmann y Gil Aluja (1986) aseveran que el uso de números borrosos triangulares en el tratamiento de la incertidumbre en la empresa es conocido desde los inicios de la incorporación de la lógica fuzzy en los problemas empresariales. Kosko (1995) explica que la lógica difusa permite utilizar conceptos relativos de la realidad, definiendo grados variables de pertenencia y siguiendo patrones de razonamiento similares a los del pensamiento humano.

Las investigaciones de los autores descritos, han centrado sus estudios en problemas de insatisfacción del cliente en diferentes empresas, por ello la teoría de la lógica borrosa entrega un aporte valioso con el propósito de tratar de dar solución a este tipo de problemas, demostrando la efectividad cuando la complejidad de los procesos es muy alto e impreciso, direccionando a una correcta toma de decisión gerencial.

\section{Metodología}

El primer paso para el desarrollo de estas técnicas, es determinar las acciones y efectos, con la finalidad de tratar de mejorar la satisfacción en el cliente del Banco Pichincha de la ciudad de Cuenca-Ecuador. Para ello se requiere el aporte de los expertos funcionarios de esta institución bancaria. Esta información se presenta a continuación: 
Tabla 1: Acciones y Efectos

\begin{tabular}{|l|l|}
\hline \multicolumn{1}{|c|}{ ACCIONES } & \multicolumn{1}{c|}{ EFECTOS } \\
\hline $\begin{array}{l}\text { Manejo eficiente de reclamos, } \\
\text { requerimientos y solicitudes de } \\
\text { clientes }\end{array}$ & $\begin{array}{l}\text { Incremento en la confianza ins- } \\
\text { titucional }\end{array}$ \\
\hline $\begin{array}{l}\text { Atención ágil que disminuya el } \\
\text { tiempo de espera de clientes }\end{array}$ & $\begin{array}{l}\text { Crecimiento de la rentabilidad } \\
\text { de la institución }\end{array}$ \\
\hline $\begin{array}{l}\text { Asesoramiento en productos y } \\
\text { servicios }\end{array}$ & $\begin{array}{l}\text { Expansión a nuevos nichos de } \\
\text { mercado }\end{array}$ \\
\hline $\begin{array}{l}\text { Trato amable en contacto con } \\
\text { el cliente }\end{array}$ & Clientes fidelizados \\
\hline $\begin{array}{l}\text { Agencias amplias y con par- } \\
\text { queos para clientes }\end{array}$ & $\begin{array}{l}\text { Generación de ventajas frente a } \\
\text { la competencia }\end{array}$ \\
\hline $\begin{array}{l}\text { Capacitación al Personal } \\
\text { Disminución de quejas, recla- } \\
\text { mos, y reprocesos }\end{array}$ \\
\hline $\begin{array}{l}\text { Disponibilidad total de canales } \\
\text { físicos y electrónicos }\end{array}$ & Aumento de venta cruzada \\
\hline $\begin{array}{l}\text { Disminución del tiempo de } \\
\text { aprobación de créditos en fá- } \\
\text { brica }\end{array}$ & Clima laboral óptimo \\
\hline $\begin{array}{l}\text { Atención al público los siete } \\
\text { días de la semana }\end{array}$ & $\begin{array}{l}\text { Mejora la imagen y reputación } \\
\text { de la organización }\end{array}$ \\
\hline $\begin{array}{l}\text { Ampliación de oferta de pro- } \\
\text { ductos servicios }\end{array}$ & Fortalecimiento de marca \\
\hline
\end{tabular}

Fuente: Autores

Con la información detallada en la tabla 1 , se puede observar que se trata de una matriz cuadrada, en donde el número de acciones coincide con el número de efectos. A continuación se desarrollan las herramientas de avanzada como la teoría del expertizaje y efectos olvidados, para encontrar la variable escondida u omitida, con la finalidad de dar solución al problema en mención, reduciendo la incertidumbre en la toma de decisión a nivel de gerencia.

La aplicación de la técnica del expertizaje, permite reducir la incertidumbre en la información obtenida. Para ello Luna, Sarmiento y Cisneros (2017) sostienen: "Se entiende por expertizaje al proceso de consulta a un grupo determinado de expertos en relación con un tema definido, con el propósito de acotar la incertidumbre" (p.5). Kaufmann y Gil-Aluja (1989) afirman: "La introducción de una valuación matizada entre 0 y 1 permite hacer intervenir niveles de verdad en la noción de incidencia. (...) Valores de 0 a 1 (la llamada valuación endecadaria) (p. 26). La escala mencionada se detalla en la tabla 2.
Tabla 2: Escala endecadaria

\begin{tabular}{|c|l|}
\hline $\begin{array}{c}\text { GRADO DE } \\
\text { PRESUNCIÓN } \\
\alpha\end{array}$ & \\
\hline 0 & No tiene incidencia \\
\hline 0,1 & Tiene mínima incidencia \\
\hline 0,2 & Tiene poca incidencia \\
\hline 0,3 & Tiene algo de incidencia \\
\hline 0,4 & Tiene una influente incidencia \\
\hline 0,5 & Tiene incidencia como no tiene incidencia \\
\hline 0,6 & Tiene bastante incidencia \\
\hline 0,7 & Tiene importante incidencia \\
\hline 0,8 & Tiene mucha incidencia \\
\hline 0,9 & Tiene muchísima incidencia \\
\hline 1 & Máxima incidencia \\
\hline
\end{tabular}

Fuente: Autores

Al tener como referente a la tabla 2, se realiza la consulta a nueve expertos de la institución financiera, quienes laboran en forma directa con los clientes y a más de ello toman decisiones de orden jerárquico. Estos funcionarios indican la incidencia entre las acciones y los efectos, con esta información en forma de ejemplo se explica el resultado de la incidencia entre el "Manejo eficiente de reclamos, requerimientos y solicitudes de clientes" sobre el "Incremento en la confianza institucional", esta información resulta: Primer experto 0,8; Segundo Experto 1,0; Tercer Experto 0,9; Cuarto Experto 0,5; Quinto Experto 0,4; Sexto Experto 1,0; Séptimo Experto 0,8; Octavo Experto 0,9; Noveno Experto 0,7. De estas opiniones, un experto indico 0,4, 0,5 y 0,7, y dos expertos manifiestan 0,8, 0,9 y 1,0.

El paso siguiente se relaciona a la normalización de la frecuencia. Se refiere a la división entre los datos de la frecuencia y la cantidad de expertos consultados. En este caso (9), resultando: $1 \div 9=0,11 ; \mathrm{y}, 2 \div 9=0,22$ y así en cada opinión. Ahora se realiza la acumulación de frecuencia, iniciando por el último valor hasta obtener la unidad, a partir de ello se considera a todos los valores en adelante uno. Por último, se efectúa la suma relacionada a la acumulación de frecuencia, desde 0,1 hasta 1. Este proceso se explica a continuación:

Tabla 3: Normalización y acumulación de frecuencias

\begin{tabular}{|l|l|l|l|}
\hline $\begin{array}{c}\text { Grado de } \\
\text { Presunción } \\
\alpha\end{array}$ & Frecuencia & $\begin{array}{c}\text { Normalización } \\
\text { de la } \\
\text { Frecuencia }\end{array}$ & $\begin{array}{c}\text { Acumulación } \\
\text { de Frecuencias }\end{array}$ \\
\hline 0 & 0 & 0 & 1,0 \\
\hline 0,1 & 0 & 0 & 1,0 \\
\hline 0,2 & 0 & 0 & 1,0 \\
\hline 0,3 & 0 & 0 & 1,0 \\
\hline 0,4 & $1 / 9$ & 0,11 & 1,0 \\
\hline 0,5 & $1 / 9$ & 0,11 & 0,89 \\
\hline 0,6 & 0 & 0 & 0,78 \\
\hline 0,7 & $1 / 9$ & 0,11 & 0,78 \\
\hline 0,8 & $2 / 9$ & 0,22 & 0,69 \\
\hline 0,9 & $2 / 9$ & 0,22 & 0,44 \\
\hline 1 & $2 / 9$ & 0,22 & 0,22 \\
\hline TOTAL & 9 & 1,00 & 7,8 \\
\hline
\end{tabular}

Fuente: Autores 
El resultado de la acumulación de frecuencias, se divide entre 10 , este dato se refiere únicamente a los valores considerados dentro del grado de presunción desde 0,1 hasta 1,0 , cuyo resultado refleja: $7,8 \div 10=0,78$. De la misma manera se aplica para el resto de incidencias entre acciones y efectos, logrando obtener la siguiente matriz:

Tabla 4: Matriz de incidencia

\begin{tabular}{|c|c|c|c|c|c|c|c|c|c|c|c|}
\hline $\mathbf{E}$ & 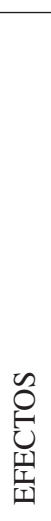 & 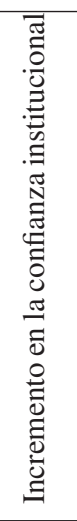 & 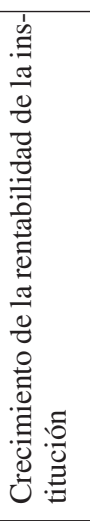 & 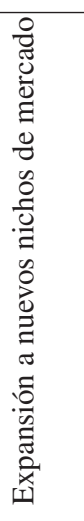 & $\begin{array}{l}0 \\
0 \\
\mathbb{0} \\
.0 \\
0 \\
0 \\
0 \\
0 \\
.00 \\
.00\end{array}$ & 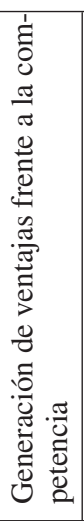 & 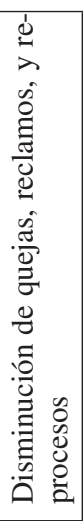 & 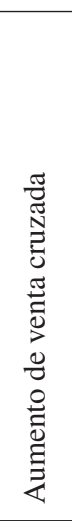 & 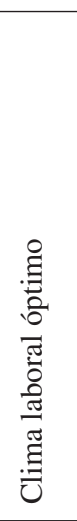 & 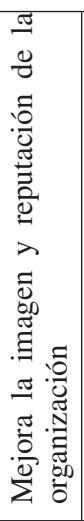 & 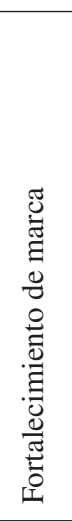 \\
\hline ACCIONES & & K & $\mathrm{L}$ & M & $\mathrm{N}$ & $\tilde{\mathrm{N}}$ & $\mathrm{O}$ & $\mathrm{P}$ & Q & $\mathrm{R}$ & $S$ \\
\hline $\begin{array}{l}\text { Manejo eficiente de reclamos, re- } \\
\text { querimientos y solicitudes de clien- } \\
\text { tes }\end{array}$ & 1 & 0,78 & 0,52 & 0,47 & 0,82 & 0,82 & 0,78 & 0,57 & 0,42 & 0,87 & 0,78 \\
\hline $\begin{array}{l}\text { Atención ágil que disminuya el } \\
\text { tiempo de espera de clientes }\end{array}$ & 2 & 0,80 & 0,74 & 0,76 & 0,82 & 0,82 & 0,78 & 0,57 & 0,47 & 0,83 & 0,66 \\
\hline $\begin{array}{l}\text { Asesoramiento en productos y ser- } \\
\text { vicios }\end{array}$ & 3 & 0,89 & 0,86 & 0,82 & 0,82 & 0,84 & 0,74 & 0,84 & 0,57 & 0,72 & 0,69 \\
\hline $\begin{array}{l}\text { Trato amable en contacto con el } \\
\text { cliente }\end{array}$ & 4 & 0,82 & 0,71 & 0,81 & 0,84 & 0,84 & 0,81 & 0,68 & 0,58 & 0,56 & 0,64 \\
\hline $\begin{array}{l}\text { Agencias amplias y con parqueos } \\
\text { para clientes }\end{array}$ & 5 & 0,56 & 0,58 & 0,52 & 0,69 & 0,69 & 0,38 & 0,39 & 0,36 & 0,68 & 0,59 \\
\hline Capacitación al Personal & 6 & 0,80 & 0,79 & 0,69 & 0,82 & 0,82 & 0,73 & 0,60 & 0,72 & 0,80 & 0,76 \\
\hline $\begin{array}{l}\text { Disponibilidad total de canales físi- } \\
\text { cos y electrónicos }\end{array}$ & 7 & 0,77 & 0,59 & 0,60 & 0,54 & 0,54 & 0,30 & 0,56 & 0,49 & 0,80 & 0,78 \\
\hline $\begin{array}{l}\text { Disminución del tiempo de aproba- } \\
\text { ción de créditos en fábrica }\end{array}$ & 8 & 0,79 & 0,87 & 0,70 & 0,79 & 0,76 & 0,62 & 0,70 & 0,53 & 0,72 & 0,83 \\
\hline $\begin{array}{l}\text { Atención al público los siete días de } \\
\text { la semana }\end{array}$ & 9 & 0,54 & 0,59 & 0,63 & 0,71 & 0,67 & 0,59 & 0,57 & 0,41 & 0,51 & 0,59 \\
\hline $\begin{array}{l}\text { Ampliación de oferta de productos } \\
\text { y servicios }\end{array}$ & 10 & 0,79 & 0,89 & 0,88 & 0,86 & 0,86 & 0,59 & 0,77 & 0,47 & 0,77 & 0,84 \\
\hline
\end{tabular}

Fuente: Elaboración propia

Partiendo de la matriz de incidencia, se desarrolla la teoría de efectos olvidados, con el cual se encuentran las variables escondidas, siendo aquellas omitidas por los expertos en la incidencia acción-efecto, en un determinado análisis. Kaufmann y Gil-Aluja (1989) establecen a través de la matriz de efectos olvidados la incidencia o números borrosos con una valoración de $[0,1]$ determinada en una escala semántica o endecadaria, siendo a 1,0 la máxima importancia y 0 sin importancia. La aplicación de esta herramienta de avanzada de la lógica difusa se detalla a continuación:
Como primer paso en la aplicación de esta herramienta, está la convolución max-min. Consiste en hallar el número mayor dentro de una sucesión de números menores, estos son producto de la comparación de filas con columnas de una matriz establecida. Para esta investigación se determina una matriz cuadrada, en donde el número de filas referente a las acciones coincide con el número de columnas relacionadas a los efectos, por ello se debe convolucionarse entre sí misma, realizado este proceso se obtiene la matriz llamada "F". Se explica como modelo la convolución entre la fila 1 con la columna $\mathrm{K}$ : 
Para 1-K:

$(111 \curlywedge K 11) \curlyvee(112 \curlywedge K 21) \curlyvee(113 \curlywedge K 31) \curlyvee(114 \curlywedge K 41) \curlyvee(115 \curlywedge K 51) \curlyvee(116 \curlywedge K 61) \curlyvee(117 \curlywedge K 71)$ $\curlyvee(118 \curlywedge K 81) \curlyvee(119 \curlywedge K 91) \curlyvee(1110 \curlywedge K 101)$

$(0,78 \curlywedge 0,78) \curlyvee(0,52 \curlywedge 0,80) \curlyvee(0,47 \curlywedge 0,89) \curlyvee(0,82 \curlywedge 0,82) \curlyvee(0,82 \curlywedge 0,56) \curlyvee(0,78 \curlywedge 0,80) \curlyvee(0,57 \curlywedge 0,77)$ $\curlyvee(0,42 \curlywedge 0,79) \curlyvee(0,87 \curlywedge 54) \curlyvee(0,78 \curlywedge 0,79)$

Se observa cada par formado, y se opta por el menor valor:

$$
0,78 \curlyvee 0,52 \curlyvee 0,47 \curlyvee 0,82 \curlyvee 0,56 \curlyvee 0,78 \curlyvee 0,57 \curlyvee 0,42 \curlyvee 0,54 \curlyvee 0,78
$$

De todos los resultados obtenidos, se escoge el número la matriz "F". El mismo procedimiento se efectúa para las mayor $(0,82)$, este se coloca en la intersección de 1 con $\mathrm{K}$ en demás coordenadas; la tabla 5 representa la matriz indicada.

Tabla 5: Matriz convolucionada "F"

\begin{tabular}{|c|c|c|c|c|c|c|c|c|c|c|c|}
\hline $\mathbf{F}$ & 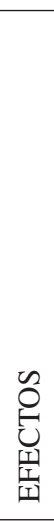 & 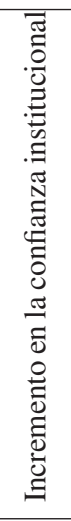 & 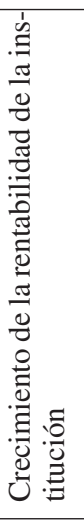 & 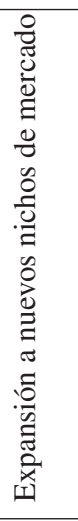 & 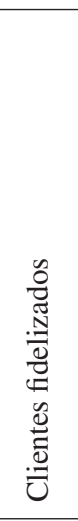 & 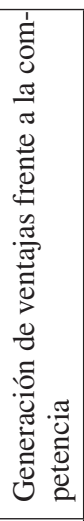 & 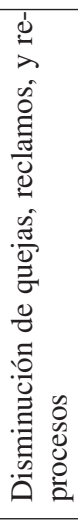 & 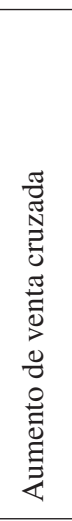 & 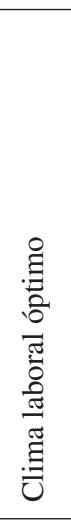 & 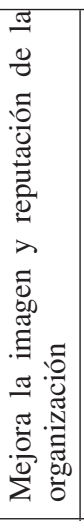 & 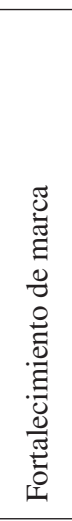 \\
\hline ACCIONES & & K & $\mathrm{L}$ & M & $\mathrm{N}$ & $\tilde{\mathrm{N}}$ & $\mathrm{O}$ & $\mathrm{P}$ & Q & $\mathrm{R}$ & S \\
\hline $\begin{array}{l}\text { Manejo eficiente de reclamos, re- } \\
\text { querimientos y solicitudes de clien- } \\
\text { tes }\end{array}$ & 1 & 0,82 & 0,78 & 0,81 & 0,82 & 0,82 & 0,81 & 0,77 & 0,72 & 0,78 & 0,78 \\
\hline $\begin{array}{l}\text { Atención ágil que disminuya el } \\
\text { tiempo de espera de clientes }\end{array}$ & 2 & 0,82 & 0,78 & 0,81 & 0,82 & 0,82 & 0,81 & 0,76 & 0,72 & 0,80 & 0,78 \\
\hline $\begin{array}{l}\text { Asesoramiento en productos y ser- } \\
\text { vicios }\end{array}$ & 3 & 0,82 & 0,82 & 0,82 & 0,82 & 0,82 & 0,81 & 0,82 & 0,72 & 0,87 & 0,78 \\
\hline $\begin{array}{l}\text { Trato amable en contacto con el } \\
\text { cliente }\end{array}$ & 4 & 0,82 & 0,81 & 0,81 & 0,84 & 0,84 & 0,81 & 0,81 & 0,72 & 0,82 & 0,78 \\
\hline $\begin{array}{l}\text { Agencias amplias y con parqueos } \\
\text { para clientes }\end{array}$ & 5 & 0,69 & 0,69 & 0,69 & 0,69 & 0,69 & 0,69 & 0,68 & 0,58 & 0,68 & 0,64 \\
\hline Capacitación al Personal & 6 & 0,82 & 0,76 & 0,81 & 0,82 & 0,82 & 0,81 & 0,76 & 0,72 & 0,80 & 0,78 \\
\hline $\begin{array}{l}\text { Disponibilidad total de canales físi- } \\
\text { cos y electrónicos }\end{array}$ & 7 & 0,78 & 0,78 & 0,78 & 0,78 & 0,78 & 0,77 & 0,77 & 0,57 & 0,77 & 0,78 \\
\hline $\begin{array}{l}\text { Disminución del tiempo de aproba- } \\
\text { ción de créditos en fábrica }\end{array}$ & 8 & 0,80 & 0,83 & 0,83 & 0,83 & 0,83 & 0,79 & 0,77 & 0,62 & 0,83 & 0,83 \\
\hline $\begin{array}{l}\text { Atención al público los siete días de } \\
\text { la semana }\end{array}$ & 9 & 0,71 & 0,71 & 0,71 & 0,71 & 0,71 & 0,71 & 0,68 & 0,59 & 0,67 & 0,64 \\
\hline $\begin{array}{l}\text { Ampliación de oferta de productos } \\
\text { y servicios }\end{array}$ & 10 & 0,88 & 0,86 & 0,84 & 0,84 & 0,84 & 0,81 & 0,84 & 0,59 & 0,83 & 0,84 \\
\hline
\end{tabular}

Fuente: Elaboración propia 
La matriz convolucionada "F", se le considera aquella que contiene las variables escondidas o efectos olvidados de primera generación, el siguiente paso es proceder a restar de esta matriz los valores de la matriz "E", respetando el orden de los cuadrantes, el resultado se expresa en valor absoluto, se explica como ejemplo de la siguiente manera; F $(1 \mathrm{~K})-\mathrm{E}$ (1k); F (1L) - E (1L); F (1M) - E (1M); y así en el mismo orden hasta completar la matriz "F-E".

Tabla 6: Resta de Matrices

\begin{tabular}{|c|c|c|c|c|c|c|c|c|c|c|c|}
\hline $\mathbf{F}$ & 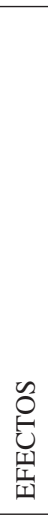 & 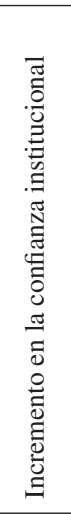 & 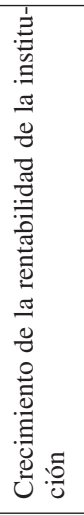 & 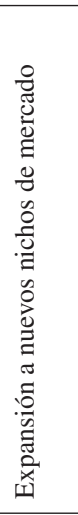 & 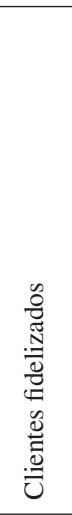 & 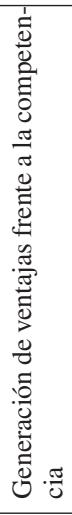 & 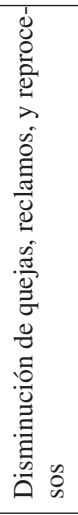 & 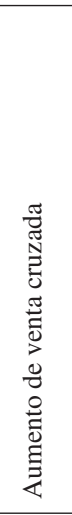 & 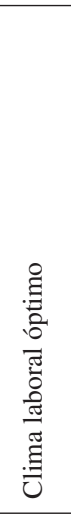 & 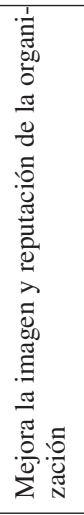 & 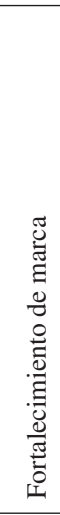 \\
\hline ACCIONES & & K & $\mathrm{L}$ & M & $\mathrm{N}$ & $\tilde{\mathrm{N}}$ & $\mathrm{O}$ & $\mathrm{P}$ & Q & $\mathrm{R}$ & $S$ \\
\hline $\begin{array}{l}\text { Manejo eficiente de reclamos, requeri- } \\
\text { mientos y solicitudes de clientes }\end{array}$ & 1 & 0,82 & 0,78 & 0,81 & 0,82 & 0,82 & 0,81 & 0,77 & 0,72 & 0,78 & 0,78 \\
\hline $\begin{array}{l}\text { Atención ágil que disminuya el tiempo } \\
\text { de espera de clientes }\end{array}$ & 2 & 0,82 & 0,78 & 0,81 & 0,82 & 0,82 & 0,81 & 0,76 & 0,72 & 0,80 & 0,78 \\
\hline Asesoramiento en productos y servicios & 3 & 0,82 & 0,82 & 0,82 & 0,82 & 0,82 & 0,81 & 0,82 & 0,72 & 0,87 & 0,78 \\
\hline Trato amable en contacto con el cliente & 4 & 0,82 & 0,81 & 0,81 & 0,84 & 0,84 & 0,81 & 0,81 & 0,72 & 0,82 & 0,78 \\
\hline $\begin{array}{l}\text { Agencias amplias y con parqueos para } \\
\text { clientes }\end{array}$ & 5 & 0,69 & 0,69 & 0,69 & 0,69 & 0,69 & 0,69 & 0,68 & 0,58 & 0,68 & 0,64 \\
\hline Capacitación al Personal & 6 & 0,82 & 0,76 & 0,81 & 0,82 & 0,82 & 0,81 & 0,76 & 0,72 & 0,80 & 0,78 \\
\hline $\begin{array}{l}\text { Disponibilidad total de canales físicos y } \\
\text { electrónicos }\end{array}$ & 7 & 0,78 & 0,78 & 0,78 & 0,78 & 0,78 & 0,77 & 0,77 & 0,57 & 0,77 & 0,78 \\
\hline $\begin{array}{l}\text { Disminución del tiempo de aprobación } \\
\text { de créditos en fábrica }\end{array}$ & 8 & 0,80 & 0,83 & 0,83 & 0,83 & 0,83 & 0,79 & 0,77 & 0,62 & 0,83 & 0,83 \\
\hline $\begin{array}{l}\text { Atención al público los siete días de la } \\
\text { semana }\end{array}$ & 9 & 0,71 & 0,71 & 0,71 & 0,71 & 0,71 & 0,71 & 0,68 & 0,59 & 0,67 & 0,64 \\
\hline $\begin{array}{l}\text { Ampliación de oferta de productos y } \\
\text { servicios }\end{array}$ & 10 & 0,88 & 0,86 & 0,84 & 0,84 & 0,84 & 0,81 & 0,84 & 0,59 & 0,83 & 0,84 \\
\hline \multicolumn{12}{|c|}{$(-)$} \\
\hline $\mathbf{E}$ & 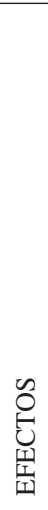 & 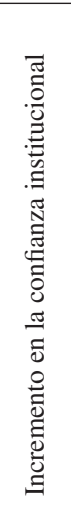 & 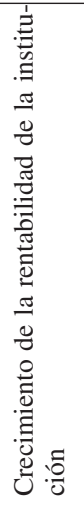 & 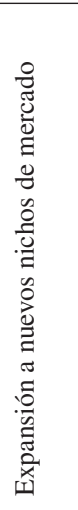 & 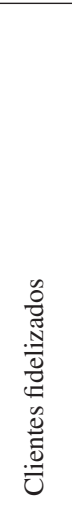 & 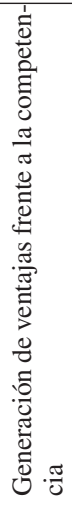 & 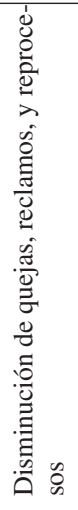 & 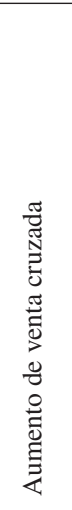 & 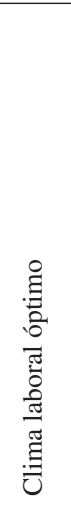 & 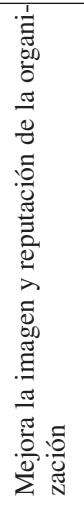 & 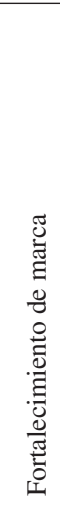 \\
\hline ACCIONES & & K & $\mathrm{L}$ & M & $\mathrm{N}$ & $\tilde{\mathrm{N}}$ & $\mathrm{O}$ & $\mathrm{P}$ & Q & $\mathrm{R}$ & $\mathrm{S}$ \\
\hline $\begin{array}{l}\text { Manejo eficiente de reclamos, requeri- } \\
\text { mientos y solicitudes de clientes }\end{array}$ & 1 & 0,78 & 0,52 & 0,47 & 0,82 & 0,82 & 0,78 & 0,57 & 0,42 & 0,87 & 0,78 \\
\hline $\begin{array}{l}\text { Atención ágil que disminuya el tiempo } \\
\text { de espera de clientes }\end{array}$ & 2 & 0,80 & 0,74 & 0,76 & 0,82 & 0,82 & 0,78 & 0,57 & 0,47 & 0,83 & 0,66 \\
\hline
\end{tabular}


Tabla 6: Resta de Matrices...(continuación)

\begin{tabular}{|c|c|c|c|c|c|c|c|c|c|c|c|}
\hline $\mathbf{E}$ & 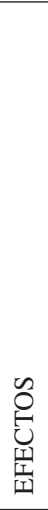 & 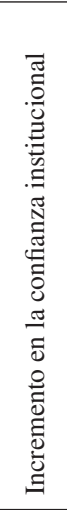 & 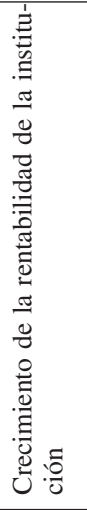 & 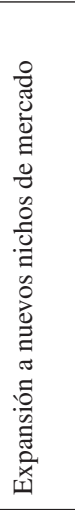 & 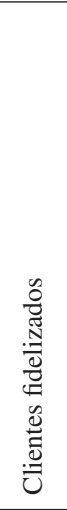 & 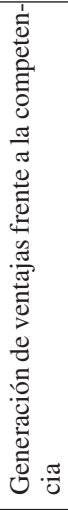 & 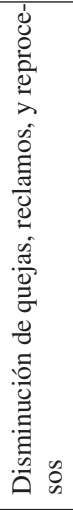 & 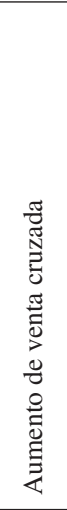 & 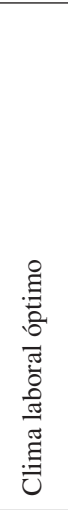 & 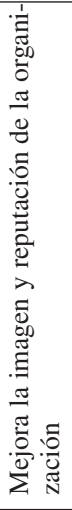 & 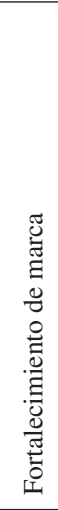 \\
\hline ACCIONES & & $\mathrm{K}$ & $\mathrm{L}$ & M & $\mathrm{N}$ & $\tilde{\mathrm{N}}$ & $\mathrm{O}$ & $\mathrm{P}$ & Q & $\mathrm{R}$ & S \\
\hline Asesoramiento en productos y servicios & 3 & 0,89 & 0,86 & 0,82 & 0,82 & 0,84 & 0,74 & 0,84 & 0,57 & 0,72 & 0,69 \\
\hline Trato amable en contacto con el cliente & 4 & 0,82 & 0,71 & 0,81 & 0,84 & 0,84 & 0,81 & 0,68 & 0,58 & 0,56 & 0,64 \\
\hline $\begin{array}{l}\text { Agencias amplias y con parqueos para } \\
\text { clientes }\end{array}$ & 5 & 0,56 & 0,58 & 0,52 & 0,69 & 0,69 & 0,38 & 0,39 & 0,36 & 0,68 & 0,59 \\
\hline Capacitación al Personal & 6 & 0,80 & 0,79 & 0,69 & 0,82 & 0,82 & 0,73 & 0,60 & 0,72 & 0,80 & 0,76 \\
\hline $\begin{array}{l}\text { Disponibilidad total de canales físicos y } \\
\text { electrónicos }\end{array}$ & 7 & 0,77 & 0,59 & 0,60 & 0,54 & 0,54 & 0,30 & 0,56 & 0,49 & 0,80 & 0,78 \\
\hline $\begin{array}{l}\text { Disminución del tiempo de aprobación } \\
\text { de créditos en fábrica }\end{array}$ & 8 & 0,79 & 0,87 & 0,70 & 0,79 & 0,76 & 0,62 & 0,70 & 0,53 & 0,72 & 0,83 \\
\hline $\begin{array}{l}\text { Atención al público los siete días de la } \\
\text { semana }\end{array}$ & 9 & 0,54 & 0,59 & 0,63 & 0,71 & 0,67 & 0,59 & 0,57 & 0,41 & 0,51 & 0,59 \\
\hline $\begin{array}{l}\text { Ampliación de oferta de productos y } \\
\text { servicios }\end{array}$ & 10 & 0,79 & 0,89 & 0,88 & 0,86 & 0,86 & 0,59 & 0,77 & 0,47 & 0,77 & 0,84 \\
\hline
\end{tabular}

Fuente: Elaboración propia

Tabla 7: Matriz de soluciones "F - E"

\begin{tabular}{|c|c|c|c|c|c|c|c|c|c|c|c|}
\hline F-E & 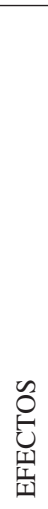 & 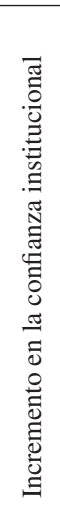 & 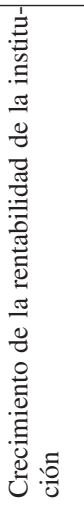 & 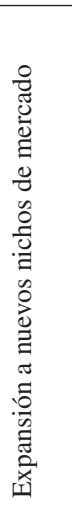 & 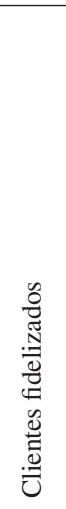 & 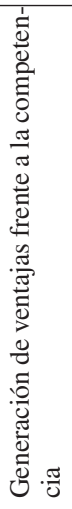 & 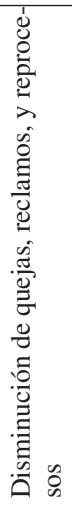 & 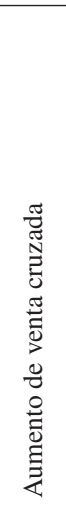 & 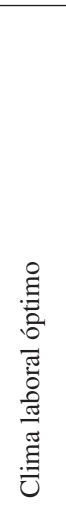 & 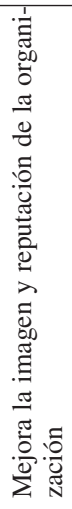 & 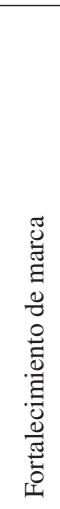 \\
\hline ACCIONES & & $\mathrm{K}$ & $\mathrm{L}$ & M & $\mathrm{N}$ & $\tilde{\mathrm{N}}$ & $\mathrm{O}$ & $\mathrm{P}$ & Q & $\mathrm{R}$ & S \\
\hline $\begin{array}{l}\text { Manejo eficiente de reclamos, requeri- } \\
\text { mientos y solicitudes de clientes }\end{array}$ & 1 & 0,04 & 0,26 & 0,34 & 0,00 & 0,00 & 0,03 & 0,20 & 0,30 & 0,09 & 0,00 \\
\hline $\begin{array}{l}\text { Atención ágil que disminuya el tiempo } \\
\text { de espera de clientes }\end{array}$ & 2 & 0,02 & 0,03 & 0,06 & 0,00 & 0,00 & 0,03 & 0,19 & 0,26 & 0,03 & 0,12 \\
\hline Asesoramiento en productos y servicios & 3 & 0,07 & 0,03 & 0,00 & 0,00 & 0,02 & 0,07 & 0,02 & 0,16 & 0,14 & 0,09 \\
\hline Trato amable en contacto con el cliente & 4 & 0,00 & 0,10 & 0,00 & 0,00 & 0,00 & 0,00 & 0,13 & 0,14 & 0,27 & 0,13 \\
\hline $\begin{array}{l}\text { Agencias amplias y con parqueos para } \\
\text { clientes }\end{array}$ & 5 & 0,13 & 0,11 & 0,17 & 0,00 & 0,00 & 0,31 & 0,29 & 0,22 & 0,00 & 0,06 \\
\hline Capacitación al Personal & 6 & 0,02 & 0,03 & 0,12 & 0,00 & 0,00 & 0,08 & 0,16 & 0,00 & 0,00 & 0,02 \\
\hline
\end{tabular}

Fuente: Elaboración propia 
Tabla 7: Matriz de soluciones “F - E”....(continuación)

\begin{tabular}{|c|c|c|c|c|c|c|c|c|c|c|c|}
\hline F-E & 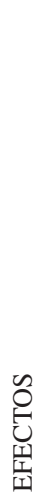 & 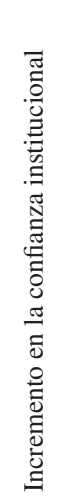 & 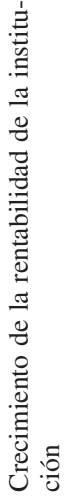 & 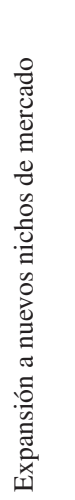 & 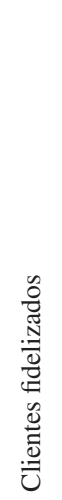 & 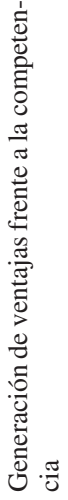 & 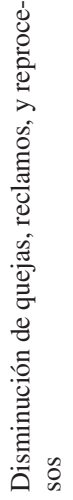 & 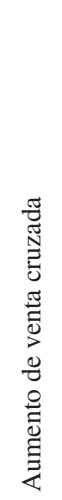 & 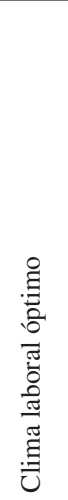 & 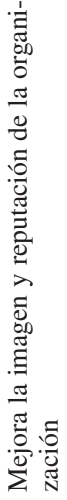 & 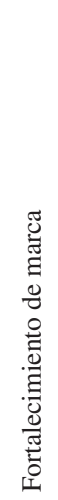 \\
\hline ACCIONES & & $\mathrm{K}$ & $\mathrm{L}$ & M & $\mathrm{N}$ & $\tilde{N}$ & $\mathrm{O}$ & $\mathrm{P}$ & Q & $\mathrm{R}$ & $\mathrm{S}$ \\
\hline $\begin{array}{l}\text { Disponibilidad total de canales físicos y } \\
\text { electrónicos }\end{array}$ & 7 & 0,01 & 0,19 & 0,18 & 0,23 & 0,23 & 0,47 & 0,21 & 0,08 & 0,03 & 0,00 \\
\hline $\begin{array}{l}\text { Disminución del tiempo de aprobación } \\
\text { de créditos en fábrica }\end{array}$ & 8 & 0,01 & 0,03 & 0,13 & 0,04 & 0,08 & 0,17 & 0,07 & 0,09 & 0,11 & 0,00 \\
\hline $\begin{array}{l}\text { Atención al público los siete días de la } \\
\text { semana }\end{array}$ & 9 & 0,17 & 0,12 & 0,08 & 0,00 & 0,04 & 0,12 & 0,11 & 0,18 & 0,16 & 0,06 \\
\hline $\begin{array}{l}\text { Ampliación de oferta de productos y } \\
\text { servicios }\end{array}$ & 10 & 0,09 & 0,03 & 0,03 & 0,01 & 0,01 & 0,22 & 0,08 & 0,12 & 0,07 & 0,00 \\
\hline
\end{tabular}

Fuente: Elaboración propia

De los resultados obtenidos en la matriz " $F-E$-, se realiza el análisis de estos valores, se eligen los más lejanos a cero o los que se aproximen a la unidad, estos se consideran con mayor alcance en relación de efectos olvidados. En la presente investigación, se estima el valor “ $\alpha$ " 0,47 y 0,34, localizados entre las intersecciones 7-O y 1-M, los valores indicados son los más cercanos a la unidad, con ello se puede decir que se tienen dos efectos olvidados o variables omitidas, entre la incidencia de la Disponibilidad total de canales físicos y electrónicos sobre Disminución de quejas, reclamos, y reprocesos; y, Manejo eficiente de reclamos, requerimientos y solicitudes de clientes sobre la Expansión a nuevos nichos de mercado. Se analiza cómo la acción incide sobre el efecto, hallando la variable que tiene incidencia de causalidad entre las mencionadas.

Se opta por el valor encontrado de " $\alpha$ " igual a 0,47 , en la intersección $(7, \mathrm{O})$ de la matriz " $\mathrm{F}$ - E", y se traslada hacia la matriz original "E". Para encontrar el efecto olvidado, se procede a realizar nuevamente la convolución max-min, comparando fila con columna de la coordenada "7, O" en la tabla 4 (matriz de incidencia), este procedimiento se presenta a continuación:

Para 7, O:

$$
\begin{aligned}
& (771 \text { 人 } O 16) \curlyvee(772 \curlywedge O 26) \curlyvee(773 \curlywedge O 36) \curlyvee(774 \curlywedge O 46) \curlyvee(775 \curlywedge O 56) \curlyvee(776 \curlywedge O 66) \curlyvee(777 \curlywedge O 76) \\
& \curlyvee(778 \text { 人 } O 86) \curlyvee(779 \curlywedge O 96) \curlyvee(7710 \curlywedge O 106) \\
& (0,77 \curlywedge 0,78) \curlyvee(0,59 \curlywedge 0,78) \curlyvee(0,60 \curlywedge 0,74) \curlyvee(0,54 \curlywedge 0,81) \curlyvee(0,54 \curlywedge 0,38) \curlyvee(0,30 \curlywedge 0,73) \curlyvee(0,56 \curlywedge 0,30) \\
& \curlyvee(0,49 \curlywedge 0,62) \curlyvee(0,80 \curlywedge 0,59) \curlyvee(0,78 \curlywedge 0,59)
\end{aligned}
$$

Se observa cada par formado, se opta por menor el valor:

$$
0,77 \curlyvee 0,59 \curlyvee 0,60 \curlyvee 0,54 \curlyvee 0,38 \curlyvee 0,73 \curlyvee 0,30 \curlyvee 0,49 \curlyvee 0,59 \curlyvee 0,59
$$

De todos los valores observados, se elige el que más se acerque a la unidad; 0,77 es el seleccionado, este representa la máxima incidencia entre la acción y el efecto de la coordenada "7, O", sobre el efecto "K". La representación gráfica explica lo enunciado: 


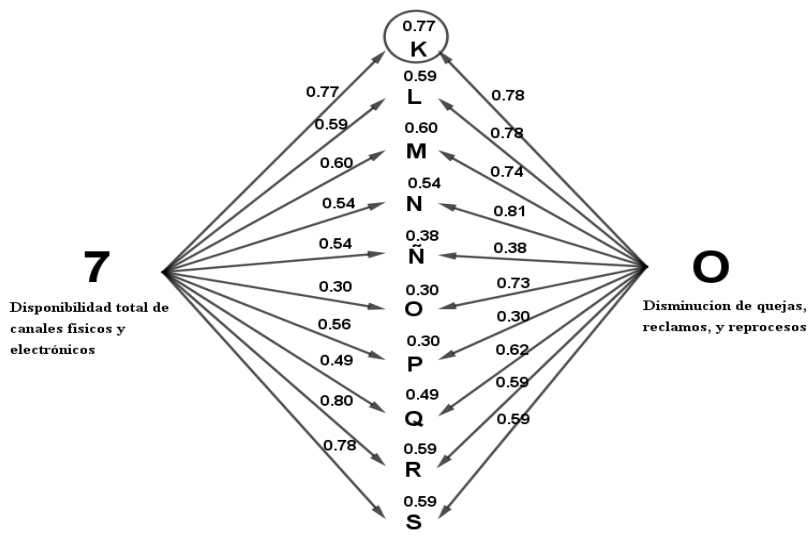

Figura 1: Incidencia de la causalidad Fuente: Autores

\section{Resultados}

Del desarrollo de estas dos teorías, tanto del expertizaje como de los efectos olvidados, el resultado obtenido es que la Disponibilidad total de canales físicos y electrónicos incide sobre la Disminución de quejas, reclamos, y reprocesos a través de la variable escondida Incremento en la confianza institucional. A partir de esta solución, los directivos deben tomar en consideración este efecto olvidado, ya que para mejorar esta variable se debe tomar en cuenta la acción a emprender, con la finalidad que el cliente sea el reflejo positivo de su satisfacción a la ciudadanía.

\section{Conclusiones y Recomendaciones}

La satisfacción del cliente, se convierte en un reto cada día de alcanzar por las diferentes organizaciones, en particular las agencias del Banco Pichincha de la ciudad de Cuenca-Ecuador, por tal razón se hace necesario que los directivos emprendan las acciones necesarias con el propósito de incrementar la confianza en la institución de acuerdo a los resultados obtenidos una vez aplicada esta herramienta. La teoría de efectos olvidados, trata de actuar sobre variables que implica relación de causalidad indirecta, con el propósito de dar un aporte importante en la toma de decisión a nivel gerencial.

En el presente estudio, se desarrolla la teoría del expertizaje con la información suministrada por los expertos funcionarios de la entidad financiera, esta técnica trata de reducir la incertidumbre o dispersión de las variables, con la finalidad de que los datos sean más ciertos y exactos, la aplicación de esta herramienta de vanguardia permite el desarrollo de la teoría de efectos olvidados, con el cual se encuentra las variables escondidas u omitidas entre las variables acciones y efectos.

Con la aplicación de estas teorías, se entrega a la gerencia de esta institución bancaria, la posibilidad de dar solución al problema de insatisfacción del cliente en el servicio que recibe, para ello se tiene que tomar en consideración los efectos olvidados de primera generación, los cuales son el camino para lograr solucionar este inconveniente, permitiendo tomar una correcta decisión, conllevando a mejorar la imagen institucional. Se recomienda a los directivos de esta entidad bancaria, considerar el desarrollo de estas herramientas que ofrece la lógica borrosa, como un aporte en la mejora de la satisfacción al cliente, abriendo la posibilidad de realizar otras investigaciones con el apoyo de esta teoría en beneficio del sector financiero.

\section{Referencias Bibliográficas}

Aguiar, F. (2004). Teoría de la decisión e incertidumbre: modelos normativos y descriptivos. Revista De Metodologia De Ciencias Sociales $N^{\circ} 8,139-160$.

Castrillón Barbosa, M. F., y Díaz Monroy, L. G. (2010). Construcción de un índice de satisfacción del clientemediante ecuaciones estructurales. Revista Apuntes del CENES, vol. XXIX, núm. 49, 31-46.

Galvis, E., y Nestor, O. (2011). edición de la satisfacción de clientes en clínicas y hospitales de Bucaramanga y área metropolitana. Scientia et Technica Año XVI, No 49, 92-97.

Gento, A., Lazzari, L., y Emilio, M. (2001). Reflexiones a cerca de las matrices de incidencia y la recuperacion de efectos olvidados. Cuadernos del CIMBAGE, 1127.

Hernández Oro, R. M., Medina León, A. A., y Hernández Pérez, G. (2014). Satisfacción del cliente en empresas de base tecnológica del sector hidráulico cubano. Ingenieria Industrial(1), 25-33.

Kaufmann, A., y Gil Aluja, J. (1986). Introduccion de la teoria de los subconjuntos borrosos a la gestion de las empresas. Barcelona España: Editorial Millandoiro.

Kaufmann, A., y Gil Aluja, J. (1989). Modelos para la investigacion de efectos olvidados. Barcelona España: Editorial Milladoiro.

Kaufmann, A., Gil Aluja, J., y Barre, R. (1987). Técnicas operativas de gestión para el tratamiento de la incertidumbre. Barcelona-España.

Kosko, B. (1995). Pensamiento Borroso: la nueva ciencia de la lógica borrosa. Barcelona España: Editorial Crítica.

Luna Altamirano, K., Sarmiento Espinoza, W., y Cisneros Quintanilla, D. (2017). Equilibrio de mercado bajo incertidumbre para la fabricación de una bota de dama. Caso Cantón Gualaceo Provincia del Azuay. Compendium, 20(39).

Pérez Campdesuñer, R., Martínez Vivar, R., y Noda Hernández, M. (2015). La satisfacción del cliente, influencia en la lealtad. Análisis del destino Holguín. Ciencias Holguín, XXI (3), 1-17.

Reig Mullor, J., y Gonzáles Carbonell, J. F. (2002). Modelo Borroso de Control de Gestión de Materiales. Revista Española de Financiación y Contabilidad, 431-459.

Rico, M., y Tinto, J. (2010). Herramientas con base en subconjuntos borrosos. Propuesta procedimental pa- 
ra aplicar expertizaje y recuperar efectos. Actualidad Contable Faces, 127-146.

Romero, G., y Romero, J. (2006). Satisfacción de clientes externos de las empresas aseguradoras en el municipio Maracaibo. Revista de Ciencias Sociales (Ve), XII (3), 563-580.

Salazar Garza, R. (2012). El peso mexicano: la gestión de cobertura del riesgo cambiario mediante la teoría de los efectos olvidados. Journal of Economics, Finance and Administrative Science, 53-73.

Tinto Arandes, J., Luna Altamirano, K. A., y Cisneros Quintanilla, D. P. (2017). Teoría de los efectos olvidados en el rescate de la imagen comercial de los artesanos del. Visión Gerencial, núm. 1, 24-42.

Vela Ortiz, D. A. (2010). La satisfacción del cliente bancario en Canadá: Diferencias entre canadienses e inmigrantes de la América Latina. Universidad \&
Empresa, 12 (18), 50-71.

Recibido: 14 de diciembre de 2018

Aceptado: 10 de marzo de 2019 